\title{
METRIC AND STRUCTURE EQUATIONS IN RELATIVISTIC CONTINUA
}

\author{
Stanislav A. Podosenov, Elena R. Men'kova
}

All-Russian Research Institute for Optical and Physical Measurements, http://www.vniiofi.ru/

Moscow 119361, Russian Federation

\section{Alexander A. Potapov}

Kotelnikov Institute of Radioengineering and Electronics, Russian Academy of Sciences, http://cplire.ru/ Moscow 125009, Russian Federation

\section{Jaykov Foukzon}

Center for Mathematical Sciences, Israel Institute of Technology, http://www.technion.ac.il/

Haifa 3200003, Israel

podosenov@mail.ru,potapov@cplire.ru,jaykovfoukzon@list.ru,e_menkova@mail.ru

Abstract. The proper expression describing physical lengths and times in arbitrary relativistic moving continua is presented. To investigate the structure equations determining the space-time geometry at specified medium characteristics are applied. In the elementary case, the geometry is the Riemannian one that does not connect with the Einstein's general relativity theory. The relativistic Born rigid uniformly accelerated reference frame realized in the Riemannian space-time is considered as an example. The relativistic Born rigid uniformly rotating reference frame without a horizon but requiring the Riemannian space-time has been constructed. The Bell inequality solution is obtained and the comparison with the Mössbauer rotor experiment is made.

Keywords: relativistic continuum, structure equations, space-time, general relativity theory, reference frame, Born rigidity, Riemannian, Einstein, Bell inequality, Mössbauer rotor

UDC 530.12, 531.134, 537.9

Bibliography - 37 references

Received 10.8.2018, accepted 12.03.2019

RENSIT, 2019, 11(2):113-124

DOI: $10.17725 /$ rensit.2019.11.113

\section{Contents}

1. INTRODUCTION (113)

2. Distance Determination MEthods IN THE LAGRANGIAN CO-MOVING NONINERTIAL REFERENCE FRAMES IN THE SPECIAL RELATIVITY THEORY (114)

3. The connection of the space-time GEOMETRY WITH CONTINUUM PROPERTIES AND FORCE FIELDS (116)

4. Relativistic Rigid ROtATiNg NONINERTIAL REFERENCE FRAMES (118)

5. Discussion OF the Mössbauer rotor EXPERIMENT (119)

6. Conclusion (121)

REFERENCES (122)

\section{INTRODUCTION}

It was shown, that in the general case flat spacetime proved to be "cramped" to describe the continuum motion in the inertial reference frame (IRF) and the transition to the noninertial reference frame (NRF) [1]. That is connected both with the force field acting on the medium particles and with the conditions imposed on the kinematic characteristics of the continuum by means of the structure equations [2-6]. These equations connect the Riemannian-Christoffel tensor and the strain velocity tensor, rotational velocity tensor and the first curvature vectors of the world lines of the medium particles. As a result, the system provided to be overdetermined and it can not be realized in the Minkowski space. This system can be solved in considering the medium motion in the Riemannian space or in the general case in the space of metric connectivity.

However if one does not constrain on the continuum characteristics and only integrates the motion equations, for example, in a flat spacetime, then no transformation of coordinates permit go beyond the scope of the flat spacetime. In applying nonholonomic transform the curvature tensor obtained from the Minkowski 
space in nonholonomic coordinates is identically zero. Provided that this zero tensor can be divided into two nonzero parts. One of them is expressed by the Christoffel symbols and other one depends on the moving medium characteristics [2-5].

\section{DISTANCE DETERMINATION} METHODS IN THE LAGRANGIAN COMOVING NONINERTIAL REFERENCE FRAMES IN THE SPECIAL RELATIVITY THEORY

In the Bell's problem [7], it was shown that the string connecting identical uniformly accelerated point rockets moving with uniform acceleration in the cosmonaut system is broken. However, its length in the inertial reference frame (IRF) does not change. Solution [7] was used to calculate the motion of an electron bunch in linear colliders in constant electric field [8]. In noninertial reference frame (NRF) frozen into a bunch or a string in the Bell's problem, the correct formula of instantaneous length does not exist. Let signature of the Minkowski space-time is (+ - - ), the Greek and Latin indices run values from 0 to 3 and from 1 to 3, respectively. Standard formula [9] for the calculation of the square element of a physical distance $d L^{2}$ by means of the spatial metric tensor

$$
\gamma_{i k}=-g_{i k}+\frac{g_{0 i} g_{0 k}}{g_{00}}
$$

is used incorrectly. The correct (in accordance with special relativity theory (SRT)) application of this formula on the hypersurface orthogonal to the world lines of the bunch particles that is the instantaneous physical space of co-moving medium observers results in the relation [10-12]

$$
L(t)=\frac{c^{2}}{a_{0}} \ln \left(\cosh \left(\frac{a_{0} L_{0}}{c^{2}}\right)+\sinh \left(\frac{a_{0} L_{0}}{c^{2}}\right) \sqrt{1+\beta^{2}}\right),
$$

where $L(t)$ is the bunch length (or the string in the Bell's problem) in the reference frame comoving to the bunch as IRF time function $t, L_{0}$ is the initial bunch (string) length, $\alpha_{0}$ is the uniform acceleration, $\beta=\alpha_{0} t / c$. The latter formula is original and unknown in scientific literature from before [10-12].

Standard calculation accordingly to formula (1) from [9] in [8], [13]

$$
L(t)=L_{0} \sqrt{1+a_{0}^{2} t^{2} / c^{2}}=\frac{L_{0}}{\sqrt{1-v^{2}(t) / c^{2}}},
$$

where the curvature of a space-like curve orthogonal to the world lines of the medium particles is neglected, at the end of speed-up in the Lagrangian co-moving NRF, results in increase of the bunch length in modern linear collider [8] in tens of thousands times. In (2) under the same conditions the bunch length increases in 1.003 times, but it does not solve the Bell's problem in principle. The approach [14] based on the calculation of the distance along the unit vector of some instantly co-moving inertial reference frame (ICIRF) from the bunch beginning to its end, results in practical nulling of the bunch length at the end of speed-up. In the Bell's problem provided $\alpha_{0} L_{0} / c^{2}<<1$ all formulae from the works above mentioned coincide.

Since many questions connected with the Bell's problem arise, we would like to give some elementary explanations. Instead of the rockets connected with a string, let us firstly consider two non-commuted identical charged particles, which interact only with the external field (the model of charged dust widely used in physics). We place these particles in the uniform electric field, let the axis coincides with the field direction. Let the second particle is located at origin, and the first particle is located at the distance $L$ from the second one. The particle interaction can be neglected as compared with the interaction with the external field. In the IFR we release these particles simultaneously at $t=0$.

The first question is. How the distance between the particles in the initial IRF at any instant of time $t$ will change? To answer the question it is necessary to consider the problem solution [9] for relativistic uniformly accelerated rectilinear motion with uniform acceleration in a proper (at the each instant of time) reference frame. Then we calculate the displacement of both particles relatively the origin of coordinates. For the first particle at the right side of the equation, we add the $L$ value (original Lagrangian coordinate). The second particle is placed at the origin of coordinates. Then we have the obvious equality $x_{1}(t)-x_{2}(t)=$ 
$x(t, L)-x(t, 0)=L=$ const. Thus, the distance between the particles remains constant. So in the initial IRF there are no any Lorentz contractions. If the distance between two particles is filled with the similar ones that turns into the dust-like bar which we call the Logunov's system [15].

It follows from the considered example that the Logunov's system is a classical rigid one. The drawback of this system is: from the viewpoint of other IRF moving relatively the initial one $t=$ const is not a simultaneity surface. Therefore, the Logunov's system is not the Lorentz-covariant one.

Let us continue the consideration of two particles (we will connect the Fermi-Walker tetrad with each particle [16]). We place the massless observer on each particle, each observer will move with constant acceleration (that is that will have a fixed first curvature vector of the world line or constant acceleration $\alpha_{0}$ in the proper NRF). Physical observer space in NRF in Fermi-Walker transport will be stretched on the Fermi triads. For our particular case, when all Fermi triads in the initial time coincide with the affine triads of Minkowski space with zero initial velocity, one of the triad reference mark always will be directed perpendicularly to one of the neighboring world lines 1 or 2 . The own world line corresponds to each particle in initial IRF. In this plane at $t=$ const the distances between the neighboring world lines remain steady and equal to $L$. However the length of perpendicular from the cross point of the line $t$ $=$ const with the world line of the second particle on the world line of the first one will not be kept in moving the particles as opposed to $L$. On the basis of the property of projection operators it follows that the distance in Fermi triads in particle motion will increase. Thus, the first particle will run from the second one. The observers on the particles will see the Lorentz elongation instead of the Lorentz contraction.

It is easy to see that if one connects two particles with a thin massless glass string then the fiber will be broken due to the Lorentz elongation but not from the Lorentz contraction. Such a viewpoint is the most famous in literature $[7,8,13]$, but it contradicts to the relativistic elasticity theory. The basis of the theory was developed by Pauli and Herglotz [17] in terms of the Born conception. Neither Lorentz contraction nor Lorentz elongation can cause the stress in a rigid body and break the string. Up to date this crude error exists in scientific literature for the explanation of the Bell's problem.

In order to generalize the classical conception of the rigid motion Born introduced the definition consistent with SRT and GRT. According to the definition the continuum motion is called rigid (in the Born's sense) if for any pair of neighboring body particles the orthogonal interval between corresponding pairs of world lines of medium particles remains constant during the motion. The difference between the classical and relativistic rigidity conditions is in the selection of spatial hypersurfaces along which distances between world lines of body particles are measured. Obviously in rigid moving hypersurfaces orthogonal to world lines in one IRF are hyperplanes orthogonal to world lines in all other IRF, that makes the Bornrigid NRF the Lorentz-covariant one as opposed to the classical rigid NRF.

According to Pauli, it is the deviation from the Born's rigidity but not the Lorentz contraction (Lorentz elongation) that results in deformations and tensions in the body.

It turns out that the standard formula for the Lorentz elongation is wrong. In non-rigid (in the Born's sense) motion, the spatial hypersurface orthogonal to the world lines is curved and this curvature is not taken in to account in the Lorentz elongation.

This solution is used to calculate the motion of electron bunch in linear colliders in constant electric field [10]. Thus, we got a paradoxical result. Particles being in completely identical conditions run from each other! Thus, the relativistic Logunov's NRF results in paradox. The main drawback of the Logunov's NRF is the absence of relativistic rigidity.

The Möller-Rindler NRF is an alternative of the Logunov NRF. The Rindler NRF is obtained from the Möller NRF [18] by simple re-designation of the Lagrangian coordinates and the transition to the dimensionless variables. The Möller NRF 
advantage is: the system is a relativistic rigid (in the Born's sense) one. Its drawback is: this NRF is not a global uniformly accelerated system and it has horizon. Each particle of the Möller medium moves with the uniform acceleration, but these accelerations are not equal each other. Therefore, the designation of the Möller transform as the transform to the uniformly accelerated NRF (as it is, for example, in Fok's book [19]) is not quite correct.

Thus, both Logunov's NRF and Möller's NRF do not eliminate all SRT paradoxes. We proved the statement that the Born rigid relativistic uniformly accelerated NRF can be realized in the Riemannian space-time which in the general case is not connected with GRT [1].

\section{THE CONNECTION OF THE SPACE- TIME GEOMETRY WITH CONTINUUM PROPERTIES AND FORCE FIELDS}

In the Newton's mechanics and SRT a mass point has zero absolute acceleration relatively IRF when the forces applied to it are absent or their vector sum is equal to zero. In GRT this rule is not fulfilled. The mass point being at rest on the surface of the gravitating sphere in accordance with GRT has the first curvature vector (4-acceleration) different from zero. Absolute acceleration is directed along external normal to the sphere and it is equal to the Newton's free fall acceleration near the surface. The support reaction force from the sphere surface moves the body from its geodesic line having zero first curvature vector only when the support reaction is absent. According to Newton, absolute acceleration of a mass point on the sphere surface is equal to zero. For weak fields Einstein's equations coincide with the Newton's theory, however the correspondence principle is not applied to absolute accelerations.

One can judge about the force field by the motion or the rest of test particles. By definition, test particles do not interact with each other, and they interact only with external field. Let test particles are identical and represent some continuum. The 4-acceleration, the strain rate tensor and the tensor of the angular velocity are the characteristics of the continuum in fourdimensional space-time. 4-acceleration is entered into the law of motion and, at the known plane metric, the field of the 4-velocity and the main medium tensors are determined by the integration of the equation of motion. Continuum in the force field specifies some reference frame (RF). For RF with the specified properties one must know the additional conditions of the main medium tensors depending on 4-velocities and 4-accelerations. For example, the demand of the rotation absence and the Born rigidity. The number of the equations for determination of the 4-velocity becomes overdetermined and the integrability conditions must be fulfilled. They are fulfilled when both 4-velocities of the medium and the metric coefficients are sought. The integrability conditions were obtained

$R_{\varepsilon \sigma, v}^{\mu} V_{\mu}=2 \nabla_{[\varepsilon} \Sigma_{\sigma] v}+2 \nabla_{[\varepsilon} \Omega_{\sigma] v}+2 \nabla_{[\varepsilon}\left(V_{\sigma]} F_{v}\right)$, (4) for which in moving continuum in fourdimensional space-time the equations are valid $\nabla_{\mu} V_{\mu}=\Sigma_{\mu v}+\Omega_{\mu v}+V_{\mu} F_{v}$,

where $V_{\mu}$ is the field of four-velocity, which satisfies the normalizing condition

$g_{\mu \nu} V^{\mu} V^{v}=1$,

$g_{\mu \nu}$ is the metric tensor in the Euler frame of reference,

$$
\begin{aligned}
& \Sigma_{\mu v}=\nabla_{(\mu} V_{v)}-V_{(\mu} F_{v)}, \\
& \Omega_{\mu v}=\nabla_{[\mu} V_{v]}-V_{[\mu} F_{v]}, \\
& F_{\mu}=V^{v} \nabla_{\nu} V_{\mu},
\end{aligned}
$$

where $\Sigma_{\mu \nu}$ is the strain-rate tensor, $\Omega_{\mu \nu}$ is the tensor of angular velocity of rotation, and $F_{\mu}$ are the first curvature vectors of the world lines of the medium particles.

One can obtain the Raychaudhuri equation [20] from the structure equation (4).

Can we repeat on their basis our NRF results? It is the technical question and does not have practical significance.

Integration of system (4-9), where $R_{\varepsilon \sigma, v}^{\mu}$ is the curvature tensor (conventionally expressed in terms of the metric tensor), yields a solution to the problem on the space-time geometry, in which NFR with a specified structure is implemented. 
Equations (4) will be referred to as structural equations for the NRF. The theorem, that the Born rigid uniformly accelerated motion is shown to be realizable in the Riemannian space, is proved [4], [21]. The structure equations are not connected with GRT but they impose additional conditions to the Einstein's equations. A theorem, according to which all static spherical symmetrical GRT solutions are compatible with the structure equations, was proven. One-dimensional solution beyond the plane infinite massive source does not exist in GRT, but the structure equation has it and it induces the metric for constant uniform static field [21].

The calculation in the Lagrangian co-moving NRF results in the metric

$$
d S^{2}=\exp \left(\frac{2 a_{0} y^{1}}{c^{2}}\right)\left(d y^{0}\right)^{2}-\left(d y^{1}\right)^{2}-\left(d y^{2}\right)^{2}-\left(d y^{3}\right)^{2},
$$

where the acceleration $\alpha_{0}$ is directed along the $y_{1}$ axis. First metric [10] was obtained in [1] and repeated in $[22,23]$. One independent component of the curvature tensor calculated by metric (10) is of the form

$$
R_{10,10}=-\frac{a_{0}^{2}}{c^{4}} \exp \left(2 a_{0} y^{1} / c^{2}\right) \text {. }
$$

The components of the Ricci tensor $R_{\beta \gamma}=g^{\alpha \gamma} R_{\alpha \beta, \gamma \delta}$ and the scalar curvature $R$ can be written as

$$
R_{00}=-R_{10,10}, R_{11}=-\frac{a_{0}^{2}}{c^{4}}, R_{10}=0, R=2 \frac{a_{0}^{2}}{c^{4}} .
$$

One can directly be convinced of the uniformly accelerated NRF (12)

$$
\begin{aligned}
& F^{1}=\frac{D V^{1}}{d S}=\frac{d V^{1}}{d S}+\Gamma_{00}^{1}\left(V^{0}\right)^{2}= \\
& =\frac{1}{g_{00}} \Gamma_{00}^{1}=-\frac{g^{11}}{2 g_{00}} \frac{\partial g_{00}}{\partial y^{1}}=\frac{a_{0}}{c^{2}} .
\end{aligned}
$$

The rest of the components of 4-accelerations are equal to zero. The metric (10) can be interpreted as the equilibrium of the probe particles in any constant uniform force field. Let the identical probe charges with the identical masses are hanged up on the weightless threads at the uniform constant electric fields. It is clear from the physical consideration that the charges are at rest relatively each other (the model of charged dust) and the tensions of all threads are identical.

Two points of view are permissible.

1. The space-time is a plane one and the sum of the forces on each charge is equal to zero.

2. The space-time is a Riemannian with the plane section and the vector of 4-acceleration is constant and it is calculated in accordance with formula (13).

Investigation of electrostatics in the Riemannian space is considered in detail in [21] and the system of the solutions of the EinsteinMaxwell equations consistent with the structure equations was obtained in $[24,25]$.

We shall develop the second point of view in accordance with GRT.

In the Riemannian geometry, the particle fixed in the field has nonzero first curvature vector (4-acceleration), and in the Minkowski space the same particle has a straight world line with zero 4-acceleration.

From the global equivalence principle, the locking of the particles in the uniform constant force field is equivalent to their occurrence in Born's rigid relativistic global uniformly accelerated NRF. In releasing the particles from the bonds, they begin to move at the starting IRF in the Minkowski space in the constant uniform electric field and the distance between the particles in IRF is not changed [2] as well as in NRF (10). In Bell's problem when starting of two point-like rockets with identical constant accelerations in the astronauts' reference frame, after the oscillation damping in the thread the world lines of the thread particles will be "parallel" to the world lines of the point-like rockets in IRF. Perfect weightless accelerometers, fixed at the weightless thread and the rockets, will show identical values. Consequently, the metric for the thread in the astronauts' reference frame coincides with (10). In NRF, the thread length is kept as well as in IRF since the initial Eulerian coordinates coincide with the Lagrange coordinates. The thread will not be broken. The paradox arises because of the standard accepted at the moment transition from IRF to NRF. 
Deduced formula (2) in SRT is correct only in the case of the standard transition from IRF to NRF.

We shall point out that the space-time is curved in the accelerated point-like rockets and the thread only in the limit of the world band. The world lines of the starting IRF particles of the Minkowski space are the straight lines parallel to the time axis and having zero first curvature vectors. From the viewpoint of any NRF, these vectors will remain zero as it is impossible to create or to zero out 4 -vectors by means of the transition from NRF to IRF and conversely with the transformation of coordinates containing the time in non-linear form. Namely such transformations of coordinates are considered by the orthodox persons as the transition from IRF to NRF and conversely. From the astronauts' viewpoint the worlds lines of the IRF particles seem not to be parallel and the medium particles of the IRF basis move on the geodetic lines relatively NRF (10). The interval element has the form $[2,3]$

$$
d S^{2}=c^{2} d t^{2}-\left(1-v^{2} / c^{2}\right)\left(d x^{1}\right)^{2}-\left(d x^{2}\right)^{2}-\left(d x^{3}\right)^{2},
$$

containing in an explicit form the Lorentz contractions and describing a synchronous RF in the Riemannian space-time. The velocity $v$ of IRF basis particles relatively NRF has the form $v$ $=c \sin \left(\alpha_{0} t / c\right)$.

\section{RELATIVISTIC RIGID ROTATING NRF}

Usually when considering the rotating disk one selects the rest-frame in which the cylindrical coordinates $r_{0}, \varphi_{0}, z_{0}, t_{0}$ are introduced and passes to the rotating reference frame $r, \varphi, z, t$ in accordance with formulae:

$r_{0}=r, \psi=\varphi_{0}+\Omega t, r_{0}=r_{2} t_{0}=t$,

where the rotational speed $\Omega$ relatively $z$ axis is considered as constant. The interval element has the form

$$
d S^{2}=\left(1-\frac{\Omega^{2} r^{2}}{c^{2}}\right) c^{2} d t^{2}-2 \Omega r^{2} d \varphi d t-d z^{2}-r^{2} d \varphi^{2}-d r^{2} .
$$

The formula holds when $r \Omega / c<1$. In [26$28]$ other velocity distributions, which restrict the linear velocity of the disk at $r \rightarrow \infty$ with the value of velocity of light $c$ and at $\Omega r / c<<1$ form $v=$ $\Omega r$, are discussed. However only usual distribution law $v=\Omega r, \Omega=$ const, satisfies to the stiffness criterion both the classic and the relativistic one (in Born's sense).

Let us determine the metric of the rigid relativistic uniformly rotating $\mathrm{NRF}$ by means of our method supposing in the formulae the strain velocity tensor $\Sigma_{\mu \nu}=0$ and demanding the constancy of the invariant characterizing the relativistic generalization of the square of the disk angular velocity $\omega$

$\Omega_{\mu \nu} \Omega^{\mu \nu}=\frac{2 \omega^{2}}{c^{2}}=$ const.

In the Lagrangian co-moving frame of reference connected with the rotating disk we have

$$
\begin{aligned}
& d S^{2}=D(r) c^{2} d t^{2}-2 P(r) c d \varphi d t-d z^{2}-r^{2} d \varphi^{2}-d r^{2} . \\
& F^{1}=\frac{1}{2 D} \frac{d D}{d r}, F^{2}=F^{3}=F^{0}=0 .
\end{aligned}
$$

Afterwards the cumbersome calculations we have two independent equations

$$
\begin{aligned}
& \frac{P}{D} \frac{d D}{d r}-\frac{d P}{d r}=-2 \frac{\omega}{c}\left(D r^{2}+P^{2}\right)^{1 / 2}, \\
& \frac{d D}{d r}=-2 \frac{\omega}{c} D P\left(D r^{2}+P^{2}\right)^{1 / 2},
\end{aligned}
$$

Condition (16) is equivalent to the constancy of the value of chronometrically invariant angular velocity vector [29] and the constancy of the value of the angular velocity in the co-moving tetrads [28].

The relativistic $\omega$ and the classic angular velocity $\Omega$ are connected by the relation

$$
\omega=\Omega\left(1-\frac{\Omega^{2} r^{2}}{c^{2}}\right)^{-1} .
$$

For metric (17) there is a steady-state solution applied in the whole sphere $0<r<\infty$ but realized in the Riemannian space-time.

The solution of the system (19), (20) in quadratures is absent. Numerical analysis showed that at $\omega r / c<<1$ metric (17) coincides with metric (15). Centripetal acceleration in the rotating NRF is determined with the formula

$$
a=c^{2} F^{1}=-\frac{\omega c P}{\sqrt{D r^{2}+P^{2}}},
$$

which at small $r$ passes to the classical one and at $r \rightarrow \infty$ gives $a=-\omega c$. The calculation of the independent nonzero components of the 
curvature tensor are cumbersome, and we omit them (see [2], [3]).

After simplifications the system $[24,25]$ is represented in the form

$$
\begin{aligned}
& \frac{d v}{d x}+\frac{v}{x}\left(1-v^{2}\right)=\left(2-v^{2}\right)\left(1-v^{2}\right), \\
& D=\exp \left(-2 \int v d x\right), v=\frac{U}{\sqrt{1+U^{2}}}, U=\frac{P}{r \sqrt{D}}, x=\frac{\omega r}{c} .
\end{aligned}
$$

Physical interpretation of function $v(x)$ means the dimensionless linear velocity of the disk. For small velocities

$$
D=\exp \left(-2 \int v d x\right)=\exp \left(-x^{2}\right)=1-x^{2}, \mathrm{P}=\frac{\omega r^{2}}{c} .
$$

that is equivalent to the classic expression. It follows from the analysis of (23) that for $x \rightarrow \infty$ the equation has the solution $v=1$. This solution is markedly differed from the classic rigid disk where the velocity field at infinity is indefinitely great. Apparently the diagram of the numerical solution (23) is resemble the diagram of the hyperbolic tangent or the deformed step function for $x>0$.

\section{DISCUSSION OF THE MÖSSBAUER ROTOR EXPERIMENT}

It is known [9] that on a revolving disk at all points, the clocks can not be identically synchronized.

From our point of view, this opinion is erroneous and it is investigated in detail in [6], [30], [31].

We refer to as "physical" space orthogonal to the world lines of medium particles. In rotating at the medium, the hypersurfaces orthogonal to particle world lines are absent [16, 29].

However, for subspaces it is not universally true. Let us divide the rotating thin disk into concentric thin hoops and consider particles located in one of them. World lines of this hoop particles in the Minkowski space (that is true for small velocities $\Omega$ ) form the congruence of the helical lines on the cylinder with radius $r$ and axis $c t$, and the congruence of space-like helical lines orthogonal to the congruence of world lines of hoop's particles will be a "physical space". This congruence is found from Pfaff's equation

$$
V_{0} d x^{0}+V_{\psi} d \psi=0, \Omega \approx \omega, \psi=\varphi+\omega t .
$$

Integrating (26), we have

$$
\begin{aligned}
& t(r, \psi)=\frac{\omega r^{2} \psi}{c^{2}},(a), \\
& t(r, \varphi)=\frac{\omega r^{2} \varphi}{c^{2}\left(1-x^{2}\right)},(b), \\
& x=\frac{\omega r}{c} .
\end{aligned}
$$

In accordance with [9] "on a revolving body at all points the clocks can not be identically synchronized. So synchronizing along a closed circuit and returning to the reference point, we obtain that the time differs from the original one by the value"

$$
\Delta t=-\frac{1}{c} \oint \frac{g_{02}}{g_{00}} d \varphi=\frac{1}{c^{2}} \oint \frac{\omega r^{2}}{\left(1-x^{2}\right)} d \varphi .
$$

We believe that the clock synchronization on the closed circuit for the rotating hoop makes sense only in a hypersurface $t=$ const. If one selects $t=$ 0 as zero readings then in all other hypersurfaces $t=$ const the clocks in different Lagrangian points of the hoop will synchronize as any hypersurface $t$ $=$ const cuts off the world lines of identical length. Formula (27 b) coincides with (28) if $\varphi=2 \pi$, , but these formulae make different sense.

We point out that formula (28) can be obtained from the solution of the Pfaff's equation in the Lagrangian co-moving RF in which the solution (27b) was obtained by integration of the Pfaff's equation

$$
\begin{aligned}
& V_{0} d x^{0}+V_{\varphi} d \varphi=0, V_{0}=g_{00} V^{0}, V_{\varphi}=g_{0 \varphi} V^{0}, \\
& V^{0}=\frac{1}{\sqrt{g_{00}}}, V^{2}=0, d t=-\frac{1}{c} \frac{g_{02}}{g_{00}} d \varphi .
\end{aligned}
$$

Whence from metric (15) taking into account (26) we have (27b). However, unlike (28) the contour integral is absent and it is changed by the integral in finite limits.

The time gap in (27b) corresponds to the time distance along the cylinder generatrix from the plane $t=0$ to the "physical" spatial similar line with $\varphi$ number. In $2 \pi$ angle the Lagrangian point $\varphi$ in "physical" space coincides with the world line of the hoop particle with $\varphi$ number.

Let us solve the following model problem. We cut a thin hollow tube from the disk center to the external radius $R_{b}$. We place a light source in 
the disk center. The tube axis has the Lagrangian coordinate $\varphi=0$, which is unchanged for all tube length. Let us define the signal frequency $v_{R}$ at the tube output if the frequency of the source signal is equal to $v_{0}$.

The world line of the end of the tube with radius $R$ forms a helical line on the surface of the cylinder with radius $R$. The cylinder axis coincides with the time line $c t$.

From the point $z=0, y=0, x=R$ coinciding with the beginning of the world line of the tube on the edge of the disk at time $t=0$ there is a "physical" spatially similar line orthogonal (in the sense of pseudo-Euclidean geometry with signature (+ - - )) to the world line of the tube end. Let at time $t=0$ the source emits a very short light pulse. In some point of time $t_{1}$ the light signal comes out of the tube end and crosses its world line. The cylinder generatrix passing through the indicated intersection point crosses the "physical" line at time $t_{2}<t_{1}$. It follows from (26), (27) that per time $t_{1}$ the end of the tube turned a corner

$$
\psi=\omega t^{1}, t_{2}(R, \psi)=X^{2} t_{1}, X^{2}=\frac{\omega^{2} R^{2}}{c^{2}} .
$$

The time distance from the "physical" line to the world line of the hoop particle at the point of its intersection with the isotropic geodesic is

$$
t_{1}-t_{2}=\Delta t=\left(1-X^{2}\right) t_{1} \text {. }
$$

For the light signal propagating inside the tube for a fixed Lagrangian coordinate $\varphi=0, z=0$, at $\Omega \approx$ $\omega$ we obtain from (15)

$$
d S^{2}=0, \frac{d r}{c \sqrt{1-x^{2}}}=d t, \frac{d r}{d t}=c \sqrt{1-x^{2}} .
$$

One can see from (31) that the coordinate speed of light tends to zero with increasing the distance from the center. However the "physical" speed of light is $d r / d t=c$. As we consider the nonrelativistic disk rotation then accurate within $\omega^{2} r^{2} / c^{2}=x^{2}<<$ 1 we obtain

$$
\int_{0}^{R} \frac{d r}{c \sqrt{1-x^{2}}}=\int_{t^{2}}^{t^{1}} d t, t_{1}-t_{2}=\left(1-X^{2}\right) t_{1}=\frac{R}{c}\left(1+X^{2} / 6\right) .
$$

The proper time is $\tau_{1}=\sqrt{1-X^{2}} t_{1}$, then

$$
\tau_{1}=\frac{R}{c} \frac{\left(1+X^{2} / 6\right)}{\sqrt{1-X^{2}}}=\tau\left(1+\frac{2 X^{2}}{3}\right) \text {. }
$$

The value $R / c \simeq \tau$ is the time of a signal propagation from the disk center to the edge in NRF on a rotating disk. One can see from (33) that $\tau_{1}>\tau$. That means that the length of the world line of the tube end is greater than the radius $R$. Formula (33) in the equivalent form using IRF time can be represented as

$$
t_{1}=t\left(1+\frac{2 X^{2}}{3}\right) \text {. }
$$

Let us carry out the following gedanken experiment to obtain the frequency change at the input $v_{0}$ and at the output $v$ of the tube. Let the radiator in the disk center generates two flashes with the $\delta t$ interval. The first flash is generated at $t=0$ and the other one $\delta t$ later. The second flash will reach the tube end at $t+\delta t$ in accordance with the IRF clock and at $t_{1}+\delta t_{1}$ accordingly to the time of the rotating disk IRF where from (34) $t_{1}=0$. Whence we have

$$
t_{1}+\delta t_{1}=(t+\delta t)\left(1+\frac{2 X^{2}}{3}\right) .
$$

Subtracting (34) from (35) and turning to frequencies, $1 / \delta t=v_{R}, 1 / \delta t_{1}=v_{0}$, we obtain

$$
v_{R}=v_{0}\left(1+\frac{2 X^{2}}{3}\right) \text {. }
$$

It is clear that instead of red shift we have violet one. It is caused by increasing the centrifugal inertial force for the observer inside the rotating tube. With increase of a distance from the radiator to the disk edge the centrifugal acceleration increases as $\omega^{2} r$.

Let us analyze the results obtained. The experimental setup of the Mössbauer rotor is described in detail in [32-34]. Our aim is the comparison with theoretical results $[32,33]$.

In deriving of formula (8) in [33] the author $[32,33]$ made a mistake. The reference to formula (10) from [35] is incorrect. We present formula (8) from [33] in our designations

$$
d \tau=d t\left(1-\frac{\omega^{2} r^{2}}{c^{2}}\right) \text {. }
$$

In accordance with [33], ([33], 8) represents increment of proper time on moving clock having the radial coordinate $r$ for $\omega r<<c$. According to 
standard viewpoint [9], true time $c$ is connected with the coordinate time $t$, for the same space point, by the relation

$$
d \tau=\sqrt{g_{00}} d t \text {. }
$$

For the Langevin's rotating disk metric (15) we have

$$
d \tau=d t \sqrt{1-\frac{\omega^{2} r^{2}}{c^{2}}} .
$$

Relations ([33], 8) and (38) are differed by the root in (38). In deriving this formula, the author [33] refers to [35] and formula (10) in it. In our designations, the formula has the form

$$
d \tau=d t-\frac{\omega r^{2} d \varphi}{c^{2}} \text {. }
$$

Formula ([33], 8) was obtained by the author from the "equality" formula

$$
d \varphi=\omega d t \text {. }
$$

The equality $\left(^{*}\right)$ is not mentioned in [33] but it is implied. We point out that $([33], 8)$ is unrelated to [35] where the author considers the Global Positioning System (GPS) and in calculations he keeps only the first order of smallness of $\omega r / c$ parameter, the second order is neglected but in $[32,33]$ it is taken into account.

Further the author [32, 33] considers the questions of radial propagation of light in NRF of a hard disk for which the increment of Lagrangian coordinates is $d \varphi=d z=0$. Thus, the reference of [33] to [35] is pointless. Instead of $([33], 8)$ the "equality" $d \tau=d t$ is obtained.

Though in [33] the formula coinciding with the Mössbauer rotor experiment was obtained [34], we consider that the theoretical results are not satisfying.

Our results also coincide with the experiment. To obtain them we used the concept of the "physical space" orthogonal to the world lines of the tube elements along which light is propagated.

\section{CONCLUSION}

There are some difficulties in describing extensive bodies in SRT [36]. The reason is misunderstanding of the concepts of a coordinate system and a reference frame. Beginning with the Einstein's works one connects the transition from IFR to NFR with the transformation of coordinates containing the time in non-linear form [19]. Therefore, at present in SRT the conventional definition of elementary rigid uniformly accelerated $\mathrm{RF}$ is absent. Fok considers that the Möller's NFR is such a system [18]. However, it is not a globally uniformly accelerated one. Each medium particle moves with a constant acceleration but these accelerations of different particles are not equal each other. In the alternative uniformly accelerated Logunov's NRF [15], all particles have identical acceleration but the relativistic Born's stiffness criterion is not fulfilled. From the starting IRF viewpoint, the Lorentz contraction between adjacent consecutive particles is absent. The Logunov's system is a classical rigid one. The enigma and difficulty of SRT is. How electrostatics describing the motion of charged dust without the initial velocity results in the violation of relativistic rigidity?

In $[5,30,31]$ the theorem is proved, that in the Minkowski space the Born-rigid and relativistic uniformly accelerated translational continuum motion is absent.

If one imposes supplementary conditions for rigidity or continuum rotations, besides the continuum motion equations, these conditions "take away" the moving medium from the flat space-time.

The obtained metric of the Born rigid globally uniformly accelerated continuum is realized in the Riemannian space-time. This metric combines the Möller's metric properties (the Born rigidity) and the Logunov's metric properties (the global uniformly acceleration). It should be noted that the proper time obtained by Einstein [37], which was called the exact time, can be obtained from the metric (10)

$$
\tau_{s}=\exp \left(\frac{a_{0} y^{1}}{c^{2}}\right) \tau,
$$

where $\tau_{\mathrm{s}}$ is the proper time for the given space point, $\tau$ is the universal time for the fixed Lagrangian particle. But Einstein dismissed the exact expression for the approximate (Möller) one.

Derived structure equations impose constraints connected with integrability of motion equations in SRT and GRT. 
The relativistic Born rigid uniformly revolving NRF without the restriction of the radius value and having at infinity the linear velocity, which is equal to the light velocity, and finite acceleration, and realized in the Riemannian space-time, is obtained. The problem concerning clock synchronization on a rotating disk was solved contrary to the statement [9] that "on a revolving body at all points the clocks can not be identically synchronized". From our viewpoint, the derivation [9] is erroneous. In formula (28), the contour in the "physical" space is unclosed. This is obvious in dividing the rotating thin disk into concentric thin hoops and considering particles located on one of them.

World lines of this hoop's particles in the Minkowski space form the congruence of the helical lines on the cylinder with radius $r$ and axis $c t$, and the congruence of space-like helical lines orthogonal to the congruence of world lines of hoop's particles will be a "physical space".

This congruence is found from Pfaff's equation. Each spacelike line is unclosed and formula (28) is inapplicable.

Time interval $t(r, \varphi)$ from $(27 b)$ corresponds to the time distance along the element of cylinder from the plane $t=0$ up to the "physical" spatially similar line $t(r, \varphi)$.

If one places identical clocks along the hoop and initially time at all clocks $t=t_{1}$ then on any hypersurface $t=$ const lengths of world lines of all clocks will be identical, that means all clocks on the hoop are synchronized. It must be from physical considerations as clocks placed at identical distances from the hoop center are in equal terms.

The solution of Bell inequality is proposed. In SRT, the Bell's paradox is not solved as according to the proved theorem $[5,31]$ in the Minkowski space conditions of relativistic rigidity and global relativistic uniform acceleration are not simultaneously satisfied.

To solve the paradox one must admit that it is impossible to realize the transition to NRF with the transformation of coordinates containing the time in non-linear form. Such transformations can not result in nonzero space-time curvature tensor.

The theory explaining the Mössbauer rotor experiment was developed.

\section{REFERENCES}

1. Podosenor SA. Geometric properties of noninertial bases in relativistic mechanics. In: Debatable Problems of Theory of Relativity and Gravitation. Ed. V. I Rodichev, N. V. Mitskevich. Moscow, Nauka Publ., 1982, 95-103.

2. Podosenov SA. Space, time and classical fields of bound structures. Moscow, Sputnik + Publ., 2000, $445 \mathrm{p}$.

3. Podosenov SA, Potapov AA, Sokolov AA. Pulse electrodynamics of wideband radio systems and fields of bound structures. Moscow, Radiotekhnika Publ., 2003, 720 p.

4. Podosenov SA. New method of field calculation in space-time of bound structures. Saarbrucken: LAP LAMBERT Academic Publishing, 2011, 352 p.

5. Podosenov SA, Potapov AA, Foukzon J, Men'kova ER. Negolonomnye, fraktalnye i svyazannye struktury $\mathrm{v}$ ralyativistskilh sploshnykh sredakh, elektrodinamike, kvantovoy mekhanike i kosmologii. V 3 tomalh. Tom 2. Silovye polya v svyasannykh i negolonomnykh strukturakh [Nonbolonomic, fractal and bound structures in relativistic continua, electrodynamics, quantum mechanics and cosmology. In three books. Book 2. Force Fields in Bound and Nonbolonomic Structures]. Moscow, LENAND Publ., 2016, $440 \mathrm{p}$.

6. Podosenov S, Foukzon J, Men'kova E. Difficulties in the Interpretation of the Einstein's Relativity Theory. Basics, Concepts, Methods. Saarbrucken, Germany: LAP LAMBERT Academic Publishing, 2017, 105 p. ISBN: 978-3-330-06799-8.

7. Bell JS. Speakable and Unspeakable in Quantum Mechanics. Cambridge University Press, 1993, 67 p.

8. Gerstein SS, Logunov AA. Zadacha JS Bella JS Bell's problem]. Elementary Particle and Nuclear Theory Physics, 1998, 29(5):1119-1132 (in Russ.).

9. Landau LD, Lifshits EM. Teoriya polya [Field theory]. Moscow, Nauka Publ., 1988, 512 p.

10. Podosenov SA, Foukzon J, Potapov AA. Zadacha Bella i issledovanie elektronnykh puchkov v lineynykh kollayderakh [Bell's problem and investigation of electron bunches 
in linear colliders]. Nelineiniy Mir, 2009, 7(8):612-622 (in Russ.).

11. Podosenov SA, Foukzon J, Potapov AA. A Study of the Motion of a Relativistic Continuous Medium. Gravitation and Cosmology, 2010, 16(4):307-312.

12. Foukzon J, Podosenov SA, Potapov AA. Relativistic Length Expansion in General Accelerated System Revisited. URL: http:// arxiv.org/pdf/0910.2298v1.

13. Ginzburg VL, Eroshenko YuN. Eshche raz o printsipe ekvivalentnosti [Once more about the equivalence principle]. UFN, 1995, 165(2):205211 (in Russ.).

14. Redzic DV. Note on Devan-Beran-Bell's Spaceship Problem. Eur. J. Phys., 2008, 29(11):11-19.

15. Logunov AA. Lectures on theory of relativity and gravitation. Modern Problem Analysis. Moscow, Nauka Publ., 1987.

16. Singe JL. Relativity: the general theory. Amsterdam, Nerth-Holland Publ., 1960, 417 p.

17. Pauli W. Theory of Relativity. Pergamon Press, 1958, $320 \mathrm{p}$.

18. Möller K. The theory of relativity. Clarendon Press Oxford, 1972, 390 p.

19. Fok VA. Teoriya prostranstua-vremeni $i$ tyagoteniya [Theory of space-time and gravitation]. Moscow, Fizmatgiz Publ., 1961, 564 p.

20. Kramer D, Stephani H, Maccallum M, Herlt E. Exact Solutions of the field equations. Ed. E. Schmutzer. Berlin: Deutscher Verlag der Wissenschaften, 1980.

21. Podosenov SA, Potapov AA, Foukzon J. Electrodynamics of a Continuous Medium in a System with Specified Structure. Physics of Wave Phenomena, 2012, 20(2):143-157.

22. Desloge EA. Nonequivalence of a Uniformly Accelerating Reference Frame and a Frame at Rest in a Uniform Gravitational Field. Am. J. Phys., 1989, 57(12):1121-1125.

23. Desloge E. A. Relativistic Motion of Free Particles in a Uniform Gravitational Field. Int. J. Theor. Phys., 1990, 29(2):193-208.

24. Podosenov SA. Struktura prostranstvavremeni i polya svyazannykh zaryadov [Spacetime Structure and Fields of Bound Charges].
Iqvestia Vuzov, Ser. Phys., 1997, 40(10):63-74 (in Russ.).

25. Podosenov SA. Space-time structure and bound-charge fields. Russian Physics Journal, 1997, 40(10):985-994. New York: Springer. ISSN 1064-8887 (Print), 1573-9228 (Online).

26. Hill EL. A Note on the Relativistic Problem of Uniform Rotation. Phys. Rev., 1946, 69(9):488-491.

27. Rosen N. Notes on Rotation and Rigid Bodies in Relativity Theory. Phys. Rev., 1947, 71(1):54-58.

28. Podosenov SA. Tetradnoe rassmotrenie vrashchatelnogo i kolebatelnogo dvizheniya $\mathrm{v}$ spetsialnoy teorii otnositelnosti [Tetrad consideration of rotary and oscillatory motion in Special Relativity Theory]. Izvestia Vuqov, Ser. Phys., 1970, 11:74-80 (in Russ.).

29. Zel'manov L. K relyativistskoy teorii anizotropnoy neodnorodnoy vselennoy [To the relativistic theory of anisotropic nonhomogeneous universe $\}$. Proc. VI Conf. on Cosmology Problems. Moscow, AN SSSR Publ., 1959, 144-174 (in Russ.).

30. Podosenov SA, Foukzon J, Men'kova ER. Structure equations of continuum in the Newton's, Maxwell's and Einstein's theories. Sciences of Europe, 2017, $2(20$ (20)):43-57.

31. Podosenov SA, Foukzon J, Men’kova ER. Structure Equations, Permitted Movement of Relativistic Continuum and Sagnac's, Erenfest's and Bell's Paradoxes. Physical Science International Journal (PSIJ), 2017, 13(2): 1-18. http:/ / www.sciencedomain.org/issue/2320; DOI: 10.9734/PSIJ/2017/30616.

32. Corda C. Interpretation of Mössbauer experiment in a rotating system: A new proof for general relativity. Annals of Physics, 2015, 355:360-366.

33. Corda C. The Mossbauer rotor experiment and the general theory of relativity. Annals of Physics, 2016, 368:258-266.

34. Kholmetskii A. L., Yarman T., Missevitch O. V., Rogozev B. I. A Mossbauer experiment in a rotating system on the second-order Doppler shift: confirmation of the corrected result by Kundig. Physica Scripta, 2009, 79(6): 065007. 
35. Ashby N. Relativity in the Global Positioning System. Living Reviews in Relativity, 2003, 6(1). Published on 28 January 2003, by the Max Planck Institute for Gravitational Physics Albert Einstein Institute, Germany. http://www.livingreviews.org/Articles / Volume6/2003-1ashby/.

36. Rodichev VI. Evolution of reference frame conception and Einstein's Programme. In book: Einstein's Collection. Ed. V. L. Ginzburg and G. I. Naan. Moscow, Nauka Publ., 1974,286-334.

37. Einstein A. Über das Relativitätsprinzip und die aus demselben gezogenen Folgerungen. Jahrb. d. Radioaktivität u. Elektronik, 1907, 4:411-462. 